Pacific Journal of Mathematics

ALMOST PERIODIC FUNCTIONS ON SEMIDIRECT
PRODUCTS OF TRANSFORMATION SEMIGROUPS 


\title{
ALMOST PERIODIC FUNCTIONS ON SEMIDIRECT PRODUCTS OF TRANSFORMATION SEMIGROUPS
}

\author{
H. D. JUNGHENN
}

\begin{abstract}
The notion of semidirect product of two transformation semigroups is introduced, and its space of almost periodic functions is expressed as a tensor product. The general techniques developed are applied to the special case of a semidirect product $S$ (ㄷ) $T$ of two semigroups. As a consequence new results are obtained on the characterization of the almost periodic compactification of $S$ () $T$ as a semidirect product of compact semigroups. A related result is the splitting of the enveloping semigroup of a semidirect product of certain flows into a semidirect product of enveloping semigroups.
\end{abstract}

o. Introduction. Let $S$ and $T$ be semitopological semigroups and $S$ (c) $T$ a semidirect product of $S$ and $T$. In an earlier paper [10] we showed that, under certain conditions, the almost periodic (a.p.) compactification $(S \text { (ㄱ) } T)^{\prime}$ of $S$ (ㄷ) $T$ is a semidirect product of the a.p. compactification of $T$ and a certain compact topological semigroup containing a dense homomorphic image of $S$. A simple corollary of this result is that the space of a.p. functions on $S$ (7) $T$ is a tensor product of the space of a.p. functions on $T$ and a subspace of a.p. functions on $S$.

In this paper we introduce the notion of semidirect product of transformation semigroups and determine exactly when its space of a.p. functions may be expressed as a tensor product in analogy with the semigroup case described above. Cast in this general setting the problem of characterizing the space of a.p. functions on a semidirect product of semigroups becomes clear, and the techniques developed lead to elegant necessary and sufficient conditions for $(S \text { () } T)^{\prime}$ to be a semidirect product. As a consequence we are able to show that $(S \text { () } T)^{\prime}$ is a semidirect product for all semitopological semigroups $S$ with identity and all semitopological groups $T$, thus generalizing results of $[10,11,12]$. The same conclusion holds if $T$ merely contains a dense subgroup. In a similar vein, but using different techniques, we show that in a wide variety of cases the enveloping semigroup of the semidirect product of two equicontinuous flows is (canonically isomorphic to) a semi- direct product of the original enveloping semigroups. 
1. Preliminaries. Let $S$ and $T$ be semitopological semigroups [1] and let $\tau: T \times S \rightarrow S$ be a separately continuous map satisfying

$$
\tau\left(t, s s^{\prime}\right)=\tau(t, s) \tau\left(t, s^{\prime}\right), \quad \tau\left(t t^{\prime}, s\right)=\tau\left(t, \tau\left(t^{\prime}, s\right)\right) .
$$

Thus $t \rightarrow \tau(t, \cdot)$ is a homomorphism from $T$ into $\operatorname{Hom}(S)$, the semigroup of all homomorphisms on $S$. We shall assume that the map $(s, t) \rightarrow s \tau\left(t, s^{\prime}\right): S \times T \rightarrow S$ is continuous for each $s^{\prime} \in S$. The semidirect product $S$ (c) $T$ of $S$ and $T$ is the topological space $S \times T$ with the multiplication ${ }^{1}$

$$
(s, t)\left(s^{\prime}, t^{\prime}\right)=\left(s \tau\left(t, s^{\prime}\right), t t^{\prime}\right) .
$$

The above conditions on $\tau$ imply that $S$ (c) $T$ is a semitopological semigroup. If $S$ (respectively $T$ ) has an identity 1 , we shall require that $\tau(t, 1)=1$ (respectively, $\tau(1, \cdot)$ is the identity mapping).

A transformation semigroup is a triple $(S, X, \pi)$ where $S$ is a semitopological semigroup, $X$ is a (Hausdorff) topological space, and $\pi: S \times X \rightarrow X$ is a separately continuous mapping (called an action) which satisfies $\pi\left(s s^{\prime}, x\right)=\pi\left(s, \pi\left(s^{\prime}, x\right)\right)$. Usually we suppress the symbol $\pi$ and write $s x$ for $\pi(s, x)$. The orbit of $x \in X$ is the set $S x=\{s x: s \in S\}$. In case $S$ has an identity 1 we require that $1 x=x$ for all $x \in X$. Note that every semitopological semigroup is a transformation semigroup, where the action is left multiplication.

If $X$ is compact, the transformation semigroup $(S, X)$ is called a flow. The enveloping semigroup of a flow $(S, X)$ is the closure in the product space $X^{X}$ of the set $\pi(S, \cdot)=\{\pi(s, \cdot): s \in S\}$ [8]; it is denoted by $E(S, X)$, or simply $E_{X}$. If the flow $(S, X)$ is equicontinuous (i.e., $\pi(S, \cdot)$ is an equicontinuous family of mappings) then $E_{X}$ is a (compact) topological semigroup with respect to the relativized product topology and composition of mappings.

Let $(S, X)$ be a transformation semigroup, $B$ a Banach space, and $C(X ; B)$ the Banach space (uniform norm) of continuous bounded $B$-valued functions on $X$. A function $f \in C(X ; B)$ is almost periodic with respect to the action of $S$ on $X$ if the set $\left\{f_{s}: s \in S\right\}$ is relatively compact in $C(X ; B)$, where $f_{s}(x)=f(s x)$. The (closed) subspace of all a.p. functions in $C(X ; B)$ is denoted by $A P(S, X ; B)$. If $B$ is the complex field then we shall suppress this symbol from the notation. Thus the usual space of a.p. functions on $S$ is denoted by $A P(S)$. $(S, X)$ is called almost periodic if $A P(S, X)=C(X)$. The reader is referred to $[1,2,4,5]$ for the general theory of a.p. functions on semigroups.

1 The definition of semidirect product given here agrees with the classical definition for groups (see, for example, [9, p. 6]), but differs from the definition given in [10, 12]. By considering "reverse" multiplication is $S, T$ and $S(\tau) T$, however, the two definitions may be shown to be equivalent. 
Let $X$ and $Y$ be topological spaces, $F$ and $G$ closed linear subspaces of $C(X)$ and $C(Y)$ respectively. For $f \in F$ and $g \in G$ define $f \otimes g \in C(X \times Y)$ by $(f \otimes g)(x, y)=f(x) g(y)$. The closed linear span in $C(X \times Y)$ of all such functions is denoted by $F \otimes G$ and is called the tensor product of $F$ and $G$. Note that $F \otimes G$ may be identified with a subspace of $C(X ; G)$ via the isometric isomorphism $h \rightarrow h^{\prime}$, where $h^{\prime}(x)=h(x, \cdot)$. More generally, let $B$ be any Banach space, and define $(f \otimes b)(x)=f(x) b(f \in F, b \in B)$. The tensor product of $F$ and $B$, denoted $F \otimes B$, is defined as the closed linear span in $C(X ; B)$ of all functions $f \otimes b$.

2. Semidirect products of transformation semigroups, Let $(S, X),(T, Y)$ be transformation semigroups and $S$ (c) $T$ a semidirect product of $S$ and $T$. Let $\sigma: T \times X \rightarrow X$ be a separately continuous mapping satisfying

$$
\sigma\left(t t^{\prime}, x\right)=\sigma\left(t, \sigma\left(t^{\prime}, x\right)\right), \quad \sigma(t, s x)=\tau(t, s) \sigma(t, x) .
$$

(In particular, $(T, X, \sigma)$ is a transformation semigroup.) We shall also require that the mapping $(s, t) \rightarrow s \sigma(t, x): S \times T \rightarrow X$ be continuous for each $x \in X$ and that $\sigma(1, \cdot)$ is the identity map if $T$ has an identity 1. The semidirect product (S ( ) $\left.T, X \times_{\sigma} Y\right)$ of $(S, X)$ and $(T, Y)$ is the transformation semigroup ( $S$ () $T, X \times Y$ ), where the action on $X \times Y$ is defined by

$$
(s, t)(x, y)=(s \sigma(t, x), t y) .
$$

Note that $\left(S \odot T, X \times{ }_{\sigma} Y\right)$ reduces to the direct product $(S \times T, X \times Y)$ if for each $t \in T, \tau(t, \cdot)$ and $\sigma(t, \cdot)$ are the identity functions.

Taking $\sigma=\tau$ one immediately sees that $S$ ( $\tau) T$, when considered as a transformation semigroup (with respect to the usual action of left multiplication), is a semidirect product of the transformation semigroups $S$ and $T$. We shall examine the a.p. properties of this kind of semidirect product in $\S 4$.

Another interesting class of semidirect products can be gotten as follows: Let $G$ be a topological group, $S$ and $T$ closed subgroups of $G$ with $S$ normal in $G, G=S T$, and $S \cap T=\{1\}$. Let $X=G / S$ and $Y=G / T$ (left coset spaces), and consider the usual actions of $S$ on $X$ and $T$ on $Y$ (e.g., if $x=s^{\prime} S$ then $s x=s s^{\prime} S$ ). Define an action $G$ on $X \times Y$ by $s t(x, y)=\left(s t x t^{-1}, t y\right)$. Then $(G, X \times Y)$ is a semidirect product of $(S, X)$ and $(T, Y)$ (where $\tau(t, s)=t s t^{-1}$ and $\left.\sigma(t, x)=t x t^{-1}\right)$.

As a third example, let $(S, X)$ and $(T, X)$ be transformation semigroups, where $S$ and $T$ are subsemigroups of a topological semigroup and $s t=t s$ for all $s \in S, t \in T$. Define an action of the direct 
product $S \times T$ on $X \times X$ by $((s, t),(x, y)) \rightarrow(s t x, t y)$. Then $(S \times T$, $X \times X)$ is a semidirect product. If the elements of $S$ and $T$ fail to commute with one another, but if $S$ and $T$ are subgroups of a topological group $G$ with $S$ normal in $G$, then the same mapping defines a semidirect product action of $S$ (c) $T$ on $X \times X$, where $\tau(t, s)=t s t^{-1}$. (In each case, $\left.\sigma(t, x)=t x.\right)$

Recall that a homomorphism from a transformation semigroup $(T, Y)$ into a transformation semigroup $(T, X)$ is a continuous map $\theta: Y \rightarrow X$ such that $\theta(t y)=t \theta(y)$ for all $t \in T, y \in Y$.

TheORem 1. Let $(S, X)$ and $(T, Y)$ be equicontinuous flows and (S (ㄱ) $T, X \times_{\sigma} Y$ ) a semidirect product. Suppose that $(T, X, \sigma)$ is a homomorphic image of $(T, Y)$. Then (S (T) $\left.T, X \times_{\sigma} Y\right)$ is equicontinuous, and if $T$ is a group then $E=E\left(\left(S\right.\right.$ (?) $\left.\left.T, X X_{\sigma} Y\right)\right)$ is canonically isomorphic (as a topological semigroup) to a semidirect product $E_{X} \odot E_{Y}$ of $E_{X}$ and $E_{Y}$.

Proof. We omit the straightforward verification that $(S$ (ㄱ) $T$, $\left.X \times_{\sigma} Y\right)$ is equicontinuous. Assume $T$ is a group, let $\theta: Y \rightarrow X$ denote the given homomorphism onto $X$, and let $\bar{\theta}: E_{Y} \rightarrow E(T, X, \sigma)$ be the unique continuous semigroup homomorphism satisfying $\bar{\theta}(\zeta)(\theta(y))=\theta(\zeta y) \quad\left(\zeta \in E_{Y}, y \in Y\right)\left[8\right.$, p. 20]. Let $\xi \in E_{X}, \zeta \in E_{Y}$ and define $\Psi(\xi, \zeta): X \times Y \rightarrow X \times Y$ by $\Psi(\xi, \zeta)(x, y)=(\xi \bar{\theta}(\zeta)(x)$, $\zeta y)$. If $\left(s_{i}\right)$ and $\left(t_{j}\right)$ are nets in $S$ and $T$, respectively, such that $s_{i} x \rightarrow \xi x(x \in X)$ and $t_{j} y \rightarrow \zeta y(y \in Y)$, then for all $y, z \in Y,\left(s_{i}, t_{j}\right)(\theta(z), y)=\left(s_{i} \theta\left(t_{j} z\right), t_{j} y\right) \rightarrow$ $(\xi \theta(\zeta z), \zeta y)=\Psi(\xi, \zeta)(\theta(z), y)$, hence $\Psi(\xi, \zeta) \in E$. A similar argument shows that every member of $E$ is of this form, hence $\Psi: E_{X} \times$ $E_{Y} \rightarrow E$ is a surjection. Note that $\Psi$ is also injective (by the surjectivity of $\theta$ and the members of $E_{Y}$ ) and continuous (since $E_{X}$ is equicontinuous). Thus $\Psi$ is a homeomorphism of $E_{X} \times E_{Y}$ onto $E$.

Next, for $\xi \in E_{X}$ and $\zeta \in E_{Y}$ define $\rho(\zeta, \xi): X \rightarrow X$ by $\rho(\zeta, \xi)=$ $\bar{\theta}(\zeta) \xi \bar{\theta}\left(\zeta^{-1}\right)$ (recalling that $T$, hence $E_{Y}$, is a group). Identifying $s \in S$ with the map it defines in $E_{X}$, and doing the same for $t \in T$, we see that for all $x \in X$,

$$
\rho(t, s) x=\bar{\theta}(t) s \bar{\theta}\left(t^{-1}\right) x=\sigma\left(t, s \sigma\left(t^{-1}, x\right)\right)=\tau(t, s) x .
$$

It follows from (1) and the equicontinuity of $(S, X)$ and $(T, Y)$ that $\rho(\zeta, \xi) x=\lim _{i, j} \tau\left(t_{j}, s_{i}\right) x(x \in X)$ whenever $\xi=\lim _{i} s_{i}$ and $\zeta=\lim _{j} t_{j}$ (pointwise limits). Therefore $\rho(\zeta, \cdot): E_{X} \rightarrow E_{X}$. It is readily verified that $\zeta \rightarrow \rho(\zeta, \cdot)$ is a homomorphism from $E_{Y}$ into Hom $\left(E_{X}\right)$, that $\rho$ is continuous, and finally that $\Psi$ is a homomorphism from $E_{X}$ (P) $E_{Y}$ onto $E$.

REMARK. If $s \in S$ and $t \in T$ are considered also as members of 
$E_{X}$ and $E_{Y}$ respectively, then

$$
\Psi(s, t)(x, y)=(s \sigma(t, x), t y)=(s, t)(x, y) .
$$

It is in this sense that the word canonical is used in the statement of Theorem 1.

The following examples show that the requirement of equicontinuity cannot in general be relaxed. In the first example, $(S, X)$ is equicontinuous, $(T, Y)$ is not. In the second example, the reverse is true.

Example 1. Let $X=Y=\{z \in C:|z|=1\}$ and $S=\{1, s\} \subset X^{X}$, where 1 is the identity mapping and $s$ is conjugation. For each positive integer $n$ define $f_{n}: X \rightarrow X$ by

$$
f_{n}\left(e^{2 \pi i r}\right)= \begin{cases}e^{\pi i(2 r)^{n}}, & 0 \leqq r \leqq 1 / 2 \\ e^{-\pi i(2-2 r)^{n}}, & 1 / 2 \leqq r \leqq 1\end{cases}
$$

Since $s$ commutes with each $f_{n}$ it commutes with every member of the group $T$ of homeomorphisms of $X$ generated by the $f_{n}$. Therefore the mapping $((s, t),(x, y)) \rightarrow(s t x, t y)$ is an action of the direct product $S \times T$ on $X \times Y$ such that $(S \times T, X \times Y)$ is a semidirect product. Let $f$ denote the pointwise limit of $\left\{f_{n}\right\}$. If $\Psi: E_{X} \times$ $E_{Y} \rightarrow E$ is any continuous mapping satisfying (2), then $\Psi(s, f)=$ $\Psi(1, f)$. Therefore $E$ cannot be canonically isomorphic to a semidirect product of $E_{X}$ and $E_{Y}$.

ExAmple 2. Let $X$ and $Y$ be as in Example 1, and take $S$ to be the group of all homeomorphisms of $X$, and $T$ the subgroup of all rotations. Define $\tau: T \times S \rightarrow S$ by $\tau(t, s)=t s t^{-1}$ and $\sigma: T \times X \rightarrow X$ by $\sigma(t, x)=t x$. Then $((s, t),(x, y)) \rightarrow(s t x, t y)$ is the action of $S$ (c) $T$ on $X \times Y$ which defines $\left(S\right.$ ( ) $T, X \times_{\sigma} Y$ ). Let $f_{n}$ be as in Example 1 , and let $g_{n}$ denote counterclockwise rotation by $\pi-1 / n$. Then $\lim _{m, n \rightarrow \infty} f_{m}\left(g_{n}(1)\right)$ does not exist, hence there can be no continuous mapping $\Psi: E_{X} \times E_{Y} \rightarrow E$ satisfying (2).

Recall that a flow $(S, X)$ is distal if $x \neq x^{\prime}$ implies the existence of a net $\left(s_{i}\right)$ in $S$ such that $\lim _{i} s_{i} x$ and $\lim _{i} s_{i} x^{\prime}$ exist and are unequal. Equivalently, $(S, X)$ is distal if and only if $E(S, X)$ is a group [8]. The following result is immediate.

Corollary 1. Let $(S, X)$ and $(T, Y)$ satisfy all of the hypotheses of the theorem. Then if $(S, X)$ is distal, so is (S () $T, X \times_{o} Y$ ).

The proximal relation in a flow $(S, X)$ is the set $P \subset X \times X$ defined as follows: $(x, y) \in P$ if and only if there exists a net $\left(s_{i}\right)$ 
in $S$ such that $\lim _{i} s_{i} x=\lim _{i} s_{i} y$. In general $P$ is only reflexive and symmetric. It is transitive if and only if $E(S, X)$ has a unique minimal left ideal [8, p. 39].

Corollary 2. Let $(S, X)$ and $(T, Y)$ satisfy all of the hypotheses of the theorem. If the proximal relation is transitive in $(S, X)$ then it is transitive in ( $S$ ( () $T, X \times_{\sigma} Y$ ).

Proof. Let $J$ be the unique minimal left ideal in $E_{X}$. We show first that $J \times E_{Y}$ is a left ideal in $E=E_{X} \odot E_{Y}$. Let $\left(\xi^{\prime}, \zeta^{\prime}\right) \in J \times E_{Y}$ and $(\xi, \zeta) \in E$. Since $\rho(\zeta, \cdot): E_{X} \rightarrow E_{X}$ is an isomorphism, $\rho(\zeta, J)=J$. Hence $(\xi, \zeta)\left(\xi^{\prime}, \zeta^{\prime}\right)=\left(\xi \rho\left(\zeta, \xi^{\prime}\right), \zeta \zeta^{\prime}\right) \in J \times E_{Y}$.

Let $K$ be any minimal left ideal contained in $J \times E_{Y}$. If $(\xi, \zeta) \in K$, then $\left(\xi \rho\left(\zeta^{-1}, \xi\right), 1\right)=\left(\xi, \zeta^{-1}\right)(\xi, \zeta) \in K$, hence the set $A=$ $\{\xi \in J:(\xi, 1) \in K\}$ is nonempty. Since $A$ is a left ideal, $A=J$. Therefore $(\xi, 1) \in K$ for every $\xi \in J$. Let $\xi \in J, \zeta \in E_{Y}$, and let $e$ be any idempotent in $J$. Then $\rho(\zeta, e)$ is an idempotent in $J$ and $J=$ $J \rho(\zeta, e)$, so $(\xi, \zeta)=(\xi \rho(\zeta, e), \zeta)=(\xi, \zeta)(e, 1) \in K$. Therefore $J \times E_{Y}$ is a minimal left ideal.

Now let $I$ be any minimal left ideal in $E$ and set $B=\left\{\xi \in E_{X}\right.$ : $(\xi, 1) \in I\}$. Then $B$ is a nonempty left ideal in $E_{X}$, hence $J \subset B$. It follows that $J \times E_{Y} \cap I \neq \phi$, so $J \times E_{Y}=I$.

3. Almost periodic functions on semidirect products. Let $(S, X),(T, Y)$ be transformation semigroups such that $S$ has an identity 1 , let $\left(S(\tau) T, X \times_{\sigma} Y\right)$ be a given semidirect product, and let

$$
F=A P\left(\left(S \subset T, X \times_{\sigma} Y\right)\right) \text {. }
$$

Define an action of $S$ (c) $T$ on $X$ by

$$
(s, t) x=s \sigma(t, x) .
$$

Clearly $A P(S \subset T, X) \otimes A P(T, Y) \subset F$. We shall determine necessary and sufficient conditions for equality to hold.

To this end we define the following auxiliary actions on $X \times Y$ :

$\alpha:(S$ (ح) $T) \times(X \times Y) \rightarrow X \times Y, \alpha((s, t),(x, y))=(s \sigma(t, x), y)$

$\beta: T \times(X \times Y) \rightarrow X \times Y, \beta(t,(x, y))=(x, t y)$

$\gamma: T \times(X \times Y) \rightarrow X \times Y, \gamma(t,(x, y))=(\sigma(t, x), y)$.

Consider the following statements:

(A) $F \subset A P(S \odot T, X \times Y, \alpha)$

(B) $F \subset A P(T, X \times Y, \beta)$

(C) $F \subset A P(T, X \times Y, \gamma)$. 
Lemma 1. If T contains a dense subgroup $G$, then (A), (B), and (C) are equivalent.

Proof. That (A) implies (C) is clear, since $A P(S$ () $T, X \times Y, \alpha) \subset$ $A P(T, X \times Y, \gamma)$.

To prove that (B) implies (A), let $f \in F$ and $\left(s_{n}\right),\left(t_{n}\right)$ sequences in $S$ and $T$ respectively. There exist subsequences $\left(p_{n}\right)$ of $\left(s_{n}\right)$ and $\left(q_{n}\right)$ of $\left(t_{n}\right)$, and $g \in F$, such that $f\left(p_{n} \sigma\left(q_{n}, x\right), q_{n} y\right) \rightrightarrows g(x, y)$ (where $\rightrightarrows$ means uniform convergence in the free variables). Since $f \in F \subset$ $A P(T, X \times Y, \beta)$ and $\bar{G}=T$, for each $n$ we may choose $r_{n} \in G$ such that

$$
\left|f\left(p_{n} \sigma\left(r_{n}, x\right), r_{n} y\right)-f\left(p_{n} \sigma\left(q_{n}, x\right), q_{n} y\right)\right|<1 / n
$$

and

$$
\left|f\left(x, r_{n} y\right)-f\left(x, q_{n} y\right)\right|<1 / n
$$

for all $x \in X, y \in Y$. Replacing $y$ in (3) and (4) by $r_{n}{ }^{-1} y$, and $x$ in (4) by $p_{n} \sigma\left(q_{n}, x\right)$, we see that $f\left(p_{n} \sigma\left(r_{n}, x\right), y\right)-g\left(x, r_{n}^{-1} y\right) \rightrightarrows 0$ and $\left|f\left(p_{n} \sigma\left(r_{n}, x\right), y\right)-f\left(p_{n} \sigma\left(q_{n}, x\right), y\right)\right|<2 / n$. Since $g \in A P(T, X \times Y, \beta)$ we may assume without loss of generality that $g\left(x, r_{n}^{-1} y\right) \rightrightarrows h(x, y)$ for some $h \in C(X \times Y)$. Then $f\left(p_{n} \sigma\left(q_{n}, x\right), y\right) \rightrightarrows h(x, y)$, so $f \in A P(S$ ( ) $T$, $X \times Y, \alpha)$. The proof that (C) implies (B) is similar.

We omit the elementary proof (essentially a diagonalization argument) of our next lemma.

Lemмa 2. Let $K$ be a relatively compact subset of a Banach space $B$, and $A$ a uniformly bounded collection of linear operators on $B$ such that $\{u x: u \in A\}$ is relatively compact in $B$ for each $x \in K$. Then each sequence $\left(u_{n}\right)$ in $A$ has a subsequence $\left(v_{n}\right)$ such that $\left(v_{n} x\right)$ converges uniformly for $x \in K$.

LEMMA 3. If $(T, Y)$ has a dense orbit then $F$ is isometric and isomorphic to $A P\left(T, Y ; A P(S\right.$ (ㄷ) $T, X)$ ) under the mapping $f \rightarrow f^{\prime}$, where $f^{\prime}(y)=(f \cdot, y)$, if and only if conditions (A) and (B) hold.

Proof. Suppose conditions (A) and (B) hold, and let $f \in F$ and $B=A P(S$ ( ) $T, X)$. Clearly, then, $f^{\prime}(y) \in B,(y \in Y)$. Claim that $f^{\prime}: Y \rightarrow C(X)$ is continuous. For let $y^{\prime} \in Y$ and $\{i\}$ the directed set of open neighborboods of $y^{\prime}$. If $f^{\prime}$ is not continuous at $y^{\prime}$, then there exist $\varepsilon>0$ and nets $\left(y_{i}\right),\left(x_{i}\right)$, with $y_{i} \in i$, such that for all $i$, $\left|f\left(x_{i}, y_{i}\right)-f\left(x_{i}, y^{\prime}\right)\right|>2 \varepsilon$. For each $i$ choose $t_{i} \in T$ such that $\left|f\left(x_{i}, t_{i} y_{0}\right)-f\left(x_{i}, y_{i}\right)\right|<\varepsilon$ and $t_{i} y_{0} \in i$, where $y_{0} \in Y$ has dense orbit. Then $t_{i} y_{0} \rightarrow y^{\prime}$, and for all $i$, 


$$
\left|f\left(x_{i}, y^{\prime}\right)-f\left(x_{i}, t_{i} y_{0}\right)\right|>\varepsilon .
$$

Choose a subnet $\left(t_{j}^{\prime}\right)$ of $\left(t_{i}\right)$ and $h \in C(X \times Y)$ such that $f\left(x, t_{j}^{\prime} y_{0}\right) \rightrightarrows$ $h\left(x, y_{0}\right)$. Then $h\left(x, y_{0}\right)=f\left(x, y^{\prime}\right)$, hence $f\left(x, t_{j}^{\prime} y_{0}\right) \rightrightarrows f\left(x, y^{\prime}\right)$, contradicting (5). Therefore $f^{\prime} \in C(Y, B)$.

To see that $f^{\prime} \in A P(T, Y ; B)$, let $\left(t_{n}\right)$ be a sequence in $T$ and choose a subsequence $\left(q_{n}\right)$ such that $f\left(x, q_{n} y\right) \rightrightarrows h(x, y)$, where $h \in$ $C(X \times Y)$. Then $\left\|f^{\prime}\left(q_{n} y\right)-h^{\prime}(y)\right\| \rightarrow 0$ uniformly in $y \in Y$.

Since $f \rightarrow f^{\prime}$ is clearly a linear isometry, it remains to show that if $g \in A P(T, Y ; B)$ and if $f(x, y)=g(y)(x)$, then $f \in F$. Let $\left(s_{n}\right)$ and $\left(t_{n}\right)$ be sequences in $S$ and $T$, respectively. For each $s \in S, t \in T$ define $u(s, t): C(X) \rightarrow C(X)$ by $u(s, t) h(x)=h(s \sigma(t, x))$. Then $\{u(s, t) g(y)$ : $s \in S, t \in T\}$ is relatively compact in $B$ for each $y \in Y$. Furthermore, $g(Y) \subset\left\{g\left(t y_{0}\right): t \in \bar{T}\right\}$, and the latter is compact in $B$. Therefore, by Lemma 2 there exists a subsequence $\left(p_{n}, q_{n}\right)$ of $\left(s_{n}, t_{n}\right)$ and $h \in C(X \times Y)$ such that $f\left(p_{n} \sigma\left(q_{n}, x\right), y\right) \rightrightarrows h(x, y)$. Since $h^{\prime} \in A P(T, Y ; B)$ we may assume without loss of generality that $h\left(x, q_{n} y\right) \rightrightarrows k(x, y)$ for some $k \in C(X \times Y)$. Thus $f\left(p_{n} \sigma\left(q_{n}, x\right), q_{n} y\right) \rightrightarrows k(x, y)$.

Conversely, if $f \rightarrow f^{\prime}$ maps $F$ onto $A P(T, Y ; B)$, then (B) obviously holds, and the argument of the previous paragraph up to the last two sentences shows that (A) also holds.

The following lemma generalizes Corollary 1(iii) of [11].

Lemma 4. Let $B$ be a Banach space. If $(T, Y)$ contains a dense orbit then $A P(T, Y ; B)=A P(T, Y) \otimes B$.

Proof. Clearly $A P(T, Y) \otimes B \subset A P(T, Y ; B)$. For each $y \in Y$ define $e(y): A P(T, Y ; B) \rightarrow B$ by $e(y) f=f(y)$. Let $L=L(A P(T, Y ; B), B)$ denote the space of bounded linear operators from $A P(T, Y ; B)$ into $B$, and give $L$ the strong operator topology. Then $e: Y \rightarrow L$ is obviously continuous. Let $Z$ denote the closure of $e(Y)$ in $L$. Since $e(Y) \subset \Pi\{f(Y): f \in A P(T, Y ; B)\}$ and each $f(Y)$ is relatively compact in $B$ (because $(T, Y)$ has a dense orbit), $Z$ is compact in $L$. Define $u(t) \in L(A P(T, Y ; B), A P(T, Y ; B))$ by $(u(t) f)(y)=f(t y)(t \in T, y \in Y)$, and let $U$ denote the strong operator closure of $u(T)$. Then an argument similar to the one for $Z$ shows that $U$ is compact in that topology [5, Theorem 3.2]. Now let $g \in C(Z)$ and let $\left(t_{n}\right)$ be any sequence in $T$. There exists a subnet $\left(q_{i}\right)$ of $\left(t_{n}\right)$ and $v \in U$ such that $u\left(q_{i}\right) \rightarrow v$ in the strong operator topology. This implies that $g \circ e\left(q_{i} y\right)=g\left(e(y) u\left(q_{i}\right)\right)$ converges uniformly to $g(e(y) v)$ in $y \in Y$. Therefore $g \circ e \in A P(T, Y)$ for every $g \in C(Z)$.

Given $\varepsilon>0$ and $f \in A P(T, Y ; B)$, let $Z_{1}, \cdots, Z_{n}$ be an open covering of $Z$ such that $\|z f-w f\|<\varepsilon$ whenever $z, w \in Z_{j}(j=1, \cdots, n)$. Let $g_{1}, \cdots, g_{n} \in C(Z)$ such that support $\left(g_{j}\right) \subset Z_{j}$ and $\sum_{j=1}^{n} g_{j}=1$. 
[7, p. 170.] Choose $z_{j} \in Z_{j}$ and set $b_{j}=z_{j} f, h_{j}=q_{j} \circ e$. Then $\left\|f-\sum_{j=1}^{n} h_{j} \otimes b_{j}\right\|<\varepsilon$, hence $f \in A P(T, Y) \otimes B$.

The following theorem is now immediate:

THeOREM 2. Let $(S, X)$ and $(T, Y)$ be transformation semigroups such that $S$ has an identity and $(T, Y)$ has a dense orbit. Then

$$
A P\left(\left(S \text { ( ) } T, X \times_{\sigma} Y\right)\right)=A P(T, Y) \otimes A P(S \text { () } T, X)
$$

if and only if conditions (A) and (B) hold.

Corollary 1. Let $(S, X)$ and $(T, Y)$ be as in Theorem 2. Then

$$
A P(S \times T, X \times Y)=A P(S, X) \otimes A P(T, Y) .
$$

Corollary 2. Let $(S, X)$ and $(T, Y)$ be as in Theorem 2, and suppose that $T$ contains a dense subgroup and $(S, X)$ has a dense orbit. If either (a) $(T, Y)$ is almost periodic, or (b) $X$ is compact and $(T, X, \sigma)$ is distal, then

$$
A P\left(\left(S \text { 두 } T, X \times_{\sigma} Y\right)\right)=A P(T, Y) \otimes A P(S \text { (ㄷ) } T, X) \text {. }
$$

Proof. By Lemma 1, it suffices to show that condition (B) holds. Let $f \in F$ and suppose that (a) holds. For each $x \in X$, then, $f(x, \cdot) \in$ $A P(T, Y)$, and since $f(X, \cdot)$ is relatively compact in $C(Y)$ (because $(S, X)$ has a dense orbit), Lemma 2 implies that $f \in A P(T, X \times Y, \beta)$.

Now suppose (b) holds. If $\left(t_{i}\right)$ is a net in $T$, there exists a subnet $\left(q_{j}\right)$ and $\xi \in E(T, X, \sigma)$ such that $\sigma\left(q_{j}, x\right) \rightarrow \xi x$ for all $x \in X$. Fix $x_{0} \in X$ and let $x^{\prime}=\xi^{-1} x_{0}$. We may assume that $f\left(\sigma\left(q_{j}, x\right), q_{j} y\right) \rightrightarrows$ $g(x, y)$ for some $g \in C(X \times Y)$. Since $(S, X)$ has a dense orbit, $x \rightarrow$ $f(x, \cdot): X \rightarrow C(Y)$ is continuous, hence $f\left(\sigma\left(q_{j}, x\right), q_{j} y\right)-f\left(x_{0}, q_{j} y\right) \rightrightarrows 0$. Therefore $f\left(x_{0}, q_{j} y\right) \rightrightarrows g\left(x^{\prime}, y\right)$, so $f\left(x_{0}, \cdot\right) \in A P(T, Y)$. Now proceed as in first paragraph.

4. Almost periodic compactification of $S$ (c) $T$. Let $T$ be a semitopological semigroup. An almost periodic compactification of $T$ is a pair $\left(T^{\prime}, \Psi\right)$, where $T^{\prime}$ is a compact topological semigroup, and $\Psi: T \rightarrow T^{\prime}$ is a continuous homomorphism with dense image such that $\Psi^{*} C\left(T^{\prime}\right)=A P(T)$ (where $\Psi^{*}: C\left(T^{\prime}\right) \rightarrow C(T)$ is the adjoint mapping $f \rightarrow f \circ \Psi)$. Almost periodic compactifications always exist and are unique up to isomorphism $[1,2,5]$.

THEOREM 3. Let $S, T$ be semitopological semigroups with identities, and $S$ (ㄷ) $T$ a semidirect product. The following are equivalent: 
(a) There exist compact topological semigroups $S^{\prime}, T^{\prime}$; continuous homomorphisms $\phi: S \rightarrow S^{\prime}, \Psi: T \rightarrow T^{\prime}$ with dense images; and a jointly continuous multiplication on the compact topological space $S^{\prime} \times T^{\prime}$ such that $\left(S^{\prime} \times T^{\prime}, \phi \times \Psi\right)$ is an a.p. compactification of $(S$ ( ) $T)$ (where $(\phi \times \Psi)(s, t)=(\phi(s), \Psi(t)))$.

(b) $A P(S(\tau) T)=A P(S \odot T, S) \otimes A P(T)$.

(c) Every member of $A P(S(\tau) T)$ is a.p. with respect to both of the actions

$$
\begin{gathered}
(S(\tau)) \times(S \times T) \longrightarrow S \times T:((s, t),(x, y)) \longrightarrow(s \tau(t, x), y) \\
T \times(S \times T) \longrightarrow S \times T:(t,(x, y)) \longrightarrow(x, t y) .
\end{gathered}
$$

If (a) holds then $\left(T^{\prime}, \Psi\right)$ is an a.p. compactification of $T$, and $S^{\prime} \times T^{\prime}$ is a semidirect product $S^{\prime} \oplus T^{\prime}$, where $\rho(\Psi(t), \phi(s))=\dot{\phi}(\tau(t, s))$.

Proof. Statements (b) and (c) are equivalent by Theorem 2.

(b) implies (a): Let $\left.((S \odot \tau))^{\prime}, \theta\right)$ denote an a.p. compactification of $S$ ( ) $T, S^{\prime}$ the spectrum of the $C^{*}$-algebra $A P(S(\tau) T, S), \dot{\phi}: S \rightarrow S^{\prime}$ the evaluation mapping $(\phi(s): f \rightarrow f(s))$, and $\left(T^{\prime}, \Psi\right)$ an a.p. compactification of $T$. By hypothesis there exists an isometric isomorphism $V: C\left(S^{\prime}\right) \otimes C\left(T^{\prime}\right) \rightarrow C\left((S \odot T)^{\prime}\right)$ such that $V(\hat{g} \otimes \hat{h})=(g \otimes h)^{\wedge}, \quad(g \in$ $A P(S(\tau) T, S), h \in A P(T))$, where $\phi^{*}(\hat{g})=g, \Psi^{*}(\hat{h})=h$ and $\theta^{*}((g \otimes$ $\left.h)^{\wedge}\right)=g \otimes h$. Since $C\left(S^{\prime}\right) \otimes C\left(T^{\prime}\right)=C\left(S^{\prime} \times T^{\prime}\right)$ there exists a homeomorphism $\eta:(S \text { ( ) } T)^{\prime} \rightarrow S^{\prime} \times T^{\prime}$ such that $\eta^{*}=V$. If $s \in S, t \in T$, $g \in A P(S(\tau) T, S)$ and $h \in A P(T)$, then $(\hat{g} \otimes \hat{h})(\eta(\theta(s, t)))=V(\hat{g} \otimes \hat{h})$ $(\theta(s, t))=(g \otimes h)^{\wedge}(\theta(s, t))=g(s) h(t)=(\hat{g} \otimes \hat{h})(\phi(s), \Psi(t)) . \quad$ It follows that $\phi \times \Psi=\eta \circ \theta$. Let $S^{\prime} \times T^{\prime}$ have the unique multiplication which makes $\eta$ a semigroup isomorphism. If $s, s^{\prime} \in S$ then $(\phi(s), \Psi(1))\left(\phi\left(s^{\prime}\right)\right.$, $\Psi(1))=\eta \circ \theta\left(s s^{\prime}, 1\right)=\left(\phi\left(s s^{\prime}\right), \Psi(1)\right)$, hence $\phi(S) \times \Psi(1)$, and therefore also $S^{\prime} \times \Psi(1)$, is a subsemigroup of $S^{\prime} \times T^{\prime}$. Thus we may define multiplication in $S^{\prime}$ so that $\phi$ and $S^{\prime}$ have the required properties.

(a) implies (b): If (a) holds then in particular $A P(S(\tau) T)=$ $(\phi \times \Psi)^{*} C\left(S^{\prime} \times T^{\prime}\right)=(\phi \times \Psi)^{*} C\left(S^{\prime}\right) \otimes C\left(T^{\prime}\right)$. We shall show that $\phi^{*} C\left(S^{\prime}\right)=A P(S$ ( ) $T, S)$. Let $g \in \phi^{*} C\left(S^{\prime}\right)$ and $\left(\left(s_{i}, t_{i}\right)\right)$ be any net in $S(\tau) T$. Choose a subnet $\left(s_{j}^{\prime}, t_{j}^{\prime}\right)$ such that $\left(\phi\left(s_{j}^{\prime}\right), \Psi\left(t_{j}^{\prime}\right)\right)$ converges to some $(x, y) \in S^{\prime} \times T^{\prime}$. Then since $S^{\prime} \times T^{\prime}$ is a compact topological semigroup, the first coordinate of $\left(\dot{\phi}\left(s_{j}^{\prime} \tau\left(t_{j}^{\prime}, s\right)\right), \Psi\left(t_{j}^{\prime}\right)\right)=\left(\phi\left(s_{j}^{\prime}\right), \Psi\left(t_{j}^{\prime}\right)\right) \times$ $(\phi(s), \Psi(1))$ converges uniformly in $s \in S$ to the first coordinate of $(x, y)(\phi(s), \Psi(1))$, and it follows that $g \in A P(S(\tau) T, S)$. Conversely, let $g \in A P(S(\tau) T, S)$. Then $g \otimes 1 \in A P(S(\tau) T)$ so there exists $h \in$ $C\left(S^{\prime} \times T^{\prime}\right)$ such that $g \otimes 1=h \circ(\phi \times \Psi)$. If $k(x)=h(x, \Psi(1))$ then $k \in C\left(S^{\prime}\right)$ and $g=k \circ \phi$. Therefore $\phi^{*} C\left(S^{\prime}\right)=A P(S$ ( ) $T, S)$. A similar argument shows that $\Psi^{*} C\left(T^{\prime}\right)=A P(T)$. Thus $A P(S \odot T)=$ $A P(S(\tau) T, S) \otimes A P(T)$, and $\left(T^{\prime}, \Psi\right)$ is an a.p. compactification of $T$. 
It remains to prove that if (a) holds, then $S^{\prime} \times T^{\prime}$ is a semidirect product. We may take $S^{\prime}$ to be the spectrum of $A P(S$ (ㄱ) $T, S)$ and $\phi: S \rightarrow S^{\prime}$ the evaluation map. For each $g \in A P(S$ (ㄷ) $T, S)$ and $t \in T$ define $v(t) g \in A P(S(\tau) T, S)$ by $(v(t) g)(s)=g((1, t) s)=g(\tau(t, s))$. Then $\delta(t, x)=v(t)^{*} x$ defines an action $\delta$ of on $S^{\prime}$ such that

$$
\delta(t, \phi(s))=\phi(\tau(t, s)) \text {. }
$$

Since this action is equicontinuous, $E=E\left(T, S^{\prime}, \delta\right)$ is a compact topological semigroup, and (6) shows that $E \subset$ Hom $(S)$. Since $t \rightarrow \delta(t, \cdot): T \rightarrow E$ is a continuous homomorphism there exists a continuous homomorphism $y \rightarrow \rho(y, \cdot): T^{\prime} \rightarrow E$ such that $\rho(\Psi(t), \cdot)=$ $\delta(t, \cdot)[5]$. In particular, $\rho(\Psi(t), \phi(s))=\phi(\tau(t, s))$. Since $\phi, \Psi$, and $\phi \times \Psi$ are homomorphisms, $\quad(\phi(s), \Psi(t))\left(\phi\left(s^{\prime}\right), \Psi\left(t^{\prime}\right)\right)=\left(\phi\left(s \tau\left(t, s^{\prime}\right)\right), \Psi(t) \Psi\left(t^{\prime}\right)\right)=$ $\left(\phi(s) \rho\left(\Psi(t), \phi\left(s^{\prime}\right)\right), \Psi(t) \Psi\left(t^{\prime}\right)\right)\left(s, s^{\prime} \in S ; t, t^{\prime} \in T\right)$, and taking nets we see that $(x, y)\left(x^{\prime}, y^{\prime}\right)=\left(x \rho\left(y, x^{\prime}\right), y y^{\prime}\right) \quad\left(x, x^{\prime} \in S^{\prime} ; y, y^{\prime} \in T^{\prime}\right)$. Therefore $S^{\prime} \times T^{\prime}=S^{\prime} @ T^{\prime}$.

The following corollary is an extension of the main results of [10].

CoROLlary 1. Let $S$ and $T$ be semitopological semigroups with identities, $S$ (ㄱ) $T$ a semidirect product, and suppose that $T$ contains a dense subgroup $G$. Then in the notation of Theorem 3, $\left(S^{\prime} @ T^{\prime \prime}\right.$, $\phi \times \psi)$ exists and is an a.p. compactification of $S(\tau) T$, and $A P(S$ (ㄷ) $T)=A P(S$ 두 $T, S) \otimes A P(T)$.

Proof. For each $t \in T$ define $U(t): A P(S$ (ㄷ) $T) \rightarrow C(S(\tau) T)$ by $U(t) f\left(s^{\prime}, t^{\prime}\right)=f\left(\tau\left(t, s^{\prime}\right), t^{\prime}\right)$. By Lemma 1 of $\S 3$, it suffices to show that $U(T) f$ is relatively norm compact for each $f \in A P(S$ (c) $T)$. Since $t \rightarrow U(t) f$ is continuous in the topology of pointwise convergence on $C(S \times T)$, it is enough to show that $U(G) f$ is relatively norm compact. For $x \in S(\tau) T$ let $L(x)$ and $R(x)$ denote respectively the left and right translation operators on $A P(S$ (c) $T)$. If $t \in G$ then

$$
U(t) f\left(s^{\prime}, t^{\prime}\right)=L(1, t) R\left(1, t^{-1}\right) R\left(1, t^{\prime}\right) f\left(s^{\prime}, 1\right)
$$

$\left(s^{\prime} \in S, t^{\prime} \in T\right)$, hence by Lemma 2 applied to $K=R(1, T) f$ and $A=$ $\left\{L(1, t) R\left(1, t^{-1}\right): t \in G\right\}$, any sequence $\left(t_{n}\right)$ of $G$ has a subsequence $\left(r_{n}\right)$ such that $U\left(r_{n}\right) f$ converges in norm.

Corollary 2. [6, 11]. Let $S, T$ be semitopological semigroups with identities and a.p. compactifications $\left(S^{\prime}, \phi\right),\left(T^{\prime}, \psi\right)$. Then $\left(S^{\prime} \times T^{\prime}, \phi \times \psi\right)$ is an a.p. compactification of the direct product $S \times T$.

Remarks. J. Berglund and P. Milnes have given an example of two left zero semigroups $S$ and $T$ for which the conclusion of 
Corollary 2 is false [3]. Also, the corresponding result for the weakly almost periodic compactification can fail even if $S$ and $T$ are locally compact abelian topological groups. (See, for example, [11], p. 663.)

\section{REFERENCES}

1. J. Berglund and K. Hofmann, Compact Semitopological Semigroups and Weakly Almost Periodic Functions, Lecture Notes in Mathematics, 42, Springer-Verlag, Berlin, 1967.

2. J. Berglund, H. Junghenn, and P. Milnes, Compact Right Topological Semigroups and Generalizations of Almost Periodicity, (in preparation).

3. J. Berglund and P. Milnes, Algebras of functions on semitopological left-groups, (to appear in Trans. Amer. Math. Soc.).

4. R. Burckel, Weakly Almost Periodic Functions on Semigroups, Gordon and Breach, New York, 1970.

5. K. deLeeuw and I. Glicksberg, Applications of almost periodic compactifications, Acta. Math., 105 (1961), 63-97.

6. - Almost periodic functions on semigroups, Acta. Math., 105 (1961), 99-140.

7. J. Dugundji, Topology, Allyn and Bacon, Boston, 1966.

8. R. Ellis, Lectures on Topological Dynamics, W. A. Benjamin, New York, 1969.

9. E. Hewitt and K. A. Ross, Abstract Harmonic Analysis I, Springer-Verlag, BerlinGoettingen-Heidelberg, 1963.

10. H. Junghenn, Almost periodic compactifications of transformation semigroups, Pacific J. Math., 57 (1975), 207-216.

11. - Tensor products of spaces of almost periodic functions, Duke Math. J., 41 (1974), 661-666.

12. M. Landstad, On the Bohr compactification of a transformation group, Math. Z.. 127 (1972), 167-178.

Received July 22, 1977.

The George Washington University

WASHINGTON, D. C. 20052 


\section{PACIFIC JOURNAL OF MATHEMATICS}

EDITORS

RICHARD ARENS (Managing Editor)

University of California

Los Angeles, California 90024

C. W. Curtis

University of Oregon

Eugene, OR 97403

C. C. MOORE

University of California

Berkeley, CA 94720
J. DUGUNDJI

Department of Mathematics University of Southern Californı Los Angeles, California 90007

R. Finn and J. Milgram Stanford University Stanford, California 94305

\section{ASSOCIATE EDITORS}

E. F. BECKENBACH

B. H. NEUMANN

F. WOLF

K. YoSHIDA

\section{SUPPORTING INSTITUTIONS}

UNIVERSITY OF BRITISH COLUMBIA
CALIFORNIA INSTITUTE OF TECHNOLOGY
UNIVERSITY OF CALIFORNIA
MONTANA STATE UNIVERSITY
UNIVERSITY OF NEVADA, RENO
NEW MEXICO STATE UNIVERSITY
OREGON STATE UNIVERSITY
UNIVERSITY OF OREGON

UNIVERSITY OF SOUTHERNEALIFORNIA STANFORD UNIVERSITY UNIVERSITY OF HAWAII UNIVERSITY OF TOKYO UNIVERSITY OF UTAH WASHINGTON STATE UNIVERSITY UNIVERSITY OF WASHINGTON 


\section{Pacific Journal of Mathematics \\ Vol. 79 , No. 1 \\ May, 1978}

Teófilo Abuabara, A remark on infinitely nuclearly differentiable

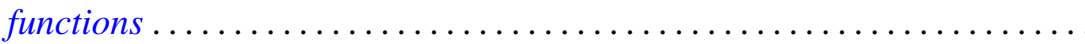

David Fenimore Anderson, Projective modules over subrings of $k[X, Y]$

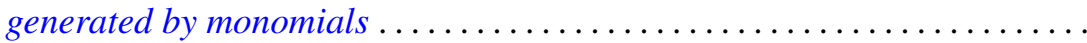

Joseph Barback and Thomas Graham McLaughlin, On the intersection of

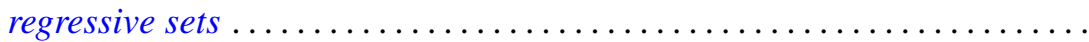

Murray Bell, John Norman Ginsburg and R. Grant Woods, Cardinal inequalities for topological spaces involving the weak Lindelof

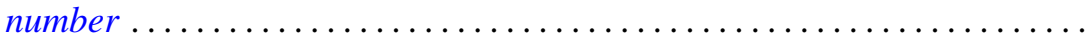

Laurence Richard Boxer, The space of ANRs of a closed surface ............

Zvonko Cerin, Homotopy properties of locally compact spaces at

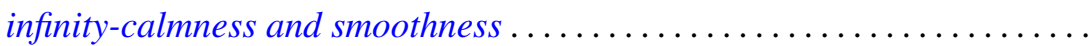

Isidor Fleischer and Ivo G. Rosenberg, The Galois connection between partial functions and relations..................................

John R. Giles, David Allan Gregory and Brailey Sims, Geometrical implications of upper semi-continuity of the duality mapping on a Banach

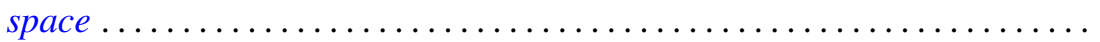

Troy Lee Hicks, Fixed-point theorems in locally convex spaces ............ Hugo Junghenn, Almost periodic functions on semidirect products of transformation semigroups ........................

Victor Kaftal, On the theory of compact operators in von Neumann algebras. II . . . .

Haynes Miller, A spectral sequence for the homology of an infinite

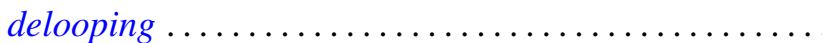

Sanford S. Miller, Petru T. Mocanu and Maxwell O. Reade, Starlike integral operators...

Stanley Stephen Page, Regular FPF rings ...............

Ghan Shyam Pandey, Multipliers for C, 1 summability of Fourier series ...

Shigeo Segawa, Bounded analytic functions on unbounded covering surfaces...

Steven Eugene Shreve, Probability measures and the C-sets of

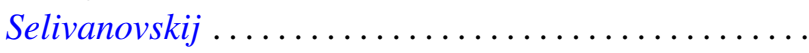

Tor Skjelbred, Combinatorial geometry and actions of compact Lie groups....

Alan Sloan, A note on exponentials of distributions.

Colin Eric Sutherland, Type analysis of the regular representation of a nonunimodular group.

Mark Phillip Thomas, Algebra homomorphisms and the functional

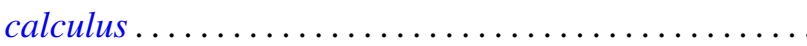

Sergio Eduardo Zarantonello, A representation of $H^{p}$-functions with

$0<p<\infty$. 